\title{
Modelo paramétrico de la fuerza de corte para el torneado de la aleación AA2024
}

\author{
J. M. SÁNCHEZ', M. MARCOS', M. Á. SEBASTIÁN², M. SÁNCHEZ', J. M. GONZÁLEZ' \\ 'Departamento de Ingeniería Mecánica y Diseño Industrial. Universidad de Cádiz. Escuela Superior de Ingeniería. 11003-Cádiz. \\ ${ }^{2}$ Departamento de Ingeniería de Construcción y Fabricación. UNED. Escuela Técnica Superior de Ingenieros Industriales. 28080-Madrid.
}

\begin{abstract}
La Fuerza de Corte, F, es uno de los parámetros de salida que puede proporcionar una mayor información sobre un determinado proceso de mecanizado. Su valor depende de una elevada cantidad de parámetros como el material y geometría de la herramienta, el material a mecanizar o los propios parámetros de corte. En este trabajo se analiza el comportamiento de la fuerza de corte en función del avance, a, y la velocidad de corte, v, en el torneado horizontal de la aleación AA2024 a una profundidad de pasada constante. A partir de este análisis, combinando las relaciones marginales $\mathrm{F}(\mathrm{v})$ y $\mathrm{F}(\mathrm{a})$, se ha podido establecer una relación paramétrica $\mathrm{F}=\mathrm{F}(\mathrm{v}, \mathrm{a})$, que permite, en primera aproximación, predecir el valor de F para unas condiciones de trabajo dependientes de las variables consideradas.
\end{abstract}

Palabras claves: Fuerza de corte, Torneado, Velocidad de corte, Avance, AA2024.

\section{Cutting Force Parametric Model for Turned AA2024 Alloy Workpieces.}

In this work, cutting force, $\mathrm{F}$, is analysed as a function of cutting speed (v) and feed (a) for dry turning processes of AA2024 (Al-Cu) alloy bars. A parametric model $\mathrm{F}=\mathrm{F}(\mathrm{v}, \mathrm{a})$ is proposed by combining $\mathrm{F}(\mathrm{v})$ and $\mathrm{F}(\mathrm{a})$ single relations. The parametric relation so obtained allows predicting de cutting force values for machining conditions that depend on those variables.

Keywords: Cutting Force, Turning, Cutting Speed, Feed, AA2024.

\section{INTRODUCCIÓN}

Un proceso de mecanizado está sujeto a un elevado número de variables, tanto de entrada (velocidad de corte, $\mathrm{v}$, avance, a, profundidad de pasada, $\mathrm{p}$, materiales, lubricación y refrigeración,....) como de salida (fuerza de corte, potencia específica, temperaturas de corte, vida de la herramienta,...) [1]. Una de las variables que puede proporcionar abundante información a la hora de evaluar uno de estos procesos es la fuerza de corte [1]. Este parámetro de salida depende de prácticamente todos los parámetros de entrada, admitiéndose [2]:

$$
\mathrm{F}=\mathrm{F}\left(\mathrm{w}_{1}, \ldots, \mathrm{w}_{\mathrm{q}^{\prime}}, \mathrm{v}, \mathrm{a}, \mathrm{p}, \mathrm{t}_{\mathrm{g} 1}, \ldots, \mathrm{t}_{\mathrm{gp}}, \mathrm{t}_{\mathrm{m} 1^{\prime}}, \ldots, \mathrm{t}_{\mathrm{m} \mathrm{r}^{\prime}} \mathrm{LR}, \mathrm{r}_{1}, \ldots, \mathrm{r}_{\mathrm{n}}\right)
$$

En esta ecuación, $\mathrm{w}_{\mathrm{i}}$ son los parámetros relacionados con el material a mecanizar, $t_{g i}$ y $t_{m h}$ los relacionados con la geometría y material de la herramienta, LR con la lubricación y refrigeración, $\mathrm{r}_{\mathrm{i}}$ con el proceso en sí, etc. Por esta razón, su control resulta complicado dadas las variables que intervienen. No obstante, si se considera un proceso concreto para un material determinado, con unas herramientas específicas y unas condiciones de lubricación fijas, la ecuación (1) deberá reducirse a una función de sólo tres variables: $p, v$ y a. Adicionalmente, si se utiliza una profundidad de pasada fija, dicha ecuación se podrá escribir,

$$
\mathrm{F}=\mathrm{F}(\mathrm{v}, \mathrm{a})
$$

facilitándose, consecuentemente, su control. En este trabajo se propone una ecuación paramétrica en la forma de la ecuación (2) para el torneado horizontal en seco de la aleación AA2024 con herramientas de TiN empleando una profundidad de pasada de $2 \mathrm{~mm}$.

\section{EXPERIMENTAL}

Los estudios de mecanizado se llevaron a cabo sobre barras cilíndricas de la aleación AA2024 (Al-4Cu-1.5Mg) sometidas a procesos de torneado horizontal en seco con herramientas recubiertas con TiN, en un centro de torneado de EMCO modelo EMCOTurn 242 TC, equipado con control numérico Emcotronic TM02. Se combinaron velocidades de corte entre 40 y $170 \mathrm{~m} / \mathrm{min}$ con avances entre 0.05 y $0.3 \mathrm{~mm} / \mathrm{rev}$. En cada ensayo se empleó una herramienta nueva con objeto de garantizar las condiciones fijadas en el proceso. Para la medición de las componentes de la fuerza de corte se empleo un dinamómetro piezoeléctrico de tres componentes KISTLER 9121, controlado por ordenador. En [3] se efectúa una descripción más detallada de este dispositivo.

\section{RESULTADOS Y DISCUSIÓN}

La fuerza en la interfase herramienta-pieza puede dividirse en tres componentes ortogonales según la velocidad de corte, $\mathrm{F}_{\mathrm{c}^{\prime}}$ el avance, $\mathrm{F}_{\mathrm{a}^{\prime}}$ y la profundidad $F_{p}$. De acuerdo con [4,5] el valor medio de $F_{c}$ una vez se alcanza el estado estacionario, es muy superior al del resto de las componentes, por lo que, en primera aproximación, puede suponerse que el valor de la fuerza total de corte es muy próximo al de la componente $\mathrm{F}_{\mathrm{c}^{\prime}}$ también denominada, por esta razón, componente principal. En este trabajo se ha estudiado el valor medio de esta componente en función de la velocidad de corte y el avance, a la que, en adelante, se denominará, simplemente, fuerza de corte y se representará por $\mathrm{F}$. Así, en la Figura 1(a) se muestra la evolución de la fuerza de corte con la velocidad de corte para distintos avances aplicados. Como puede apreciarse, de acuerdo con lo observado en [4] cuando se trabaja con herramientas $\mathrm{TiCN}$, la fuerza es creciente con la velocidad para pequeños avances, invirtiéndose la tendencia para avances mayores. Al representar F(v) en escala logarítmica, puede observarse, Figura 1(b), cómo puede proponerse un ajuste lineal entre ambas variables en dicha escala. Este hecho permite suponer un comportamiento potencial 

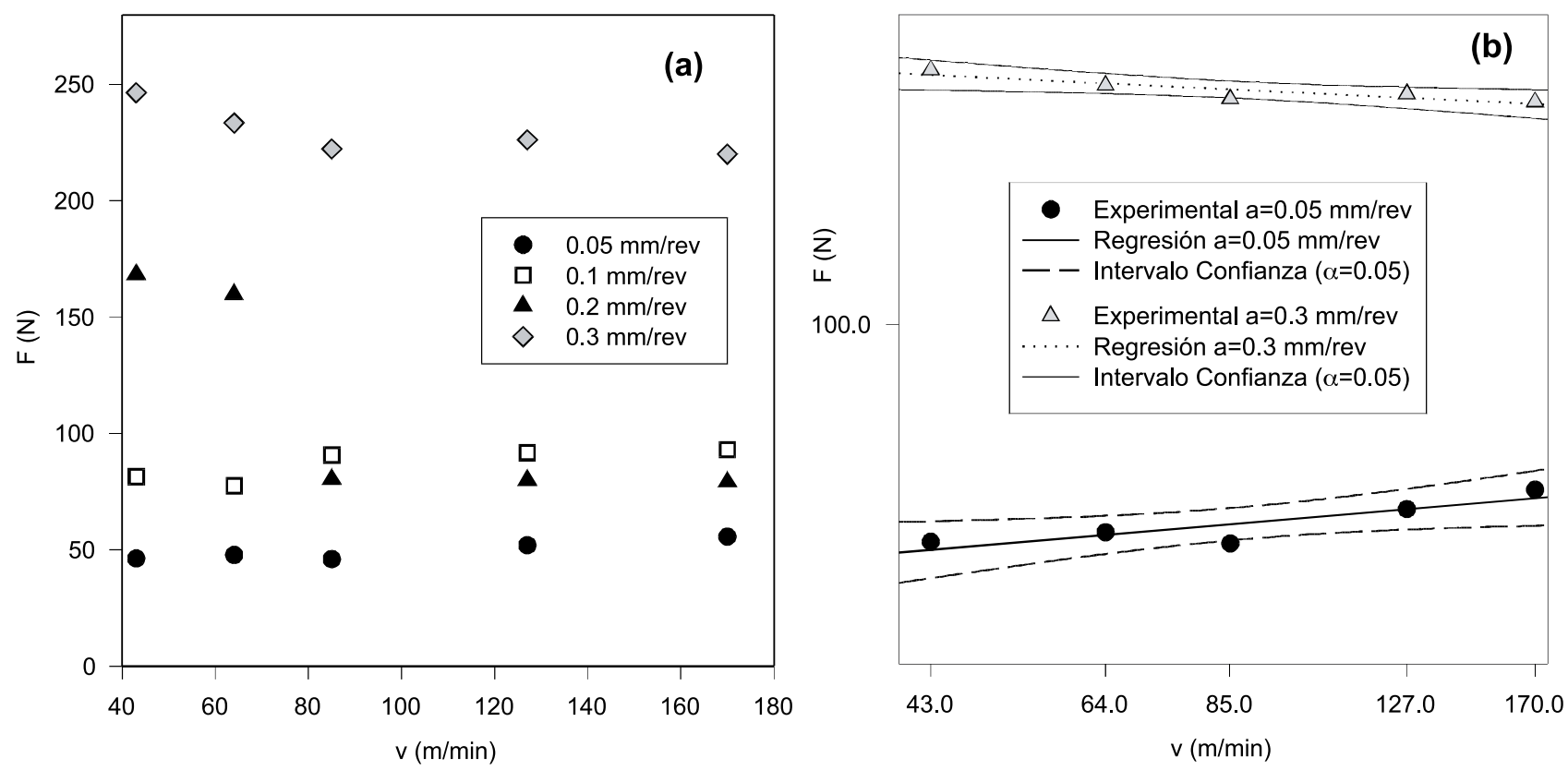

Figura 1. Evolución de la Fuerza de Corte con la velocidad para los distintos avances aplicados. (a) Escala Lineal. (b) Escala Logarítmica.

de F en función de la velocidad de corte de la forma:

$$
\mathrm{F}=\mathrm{k}_{\mathrm{a}} \cdot \mathrm{v}^{\mathrm{m}}
$$

En esta ecuación, $\mathrm{k}_{\mathrm{a}}$ y $\mathrm{m}$ dependen del avance aplicado. Al realizar el ajuste, se obtiene, en cada caso, una recta de regresión

$$
\log \mathrm{F}=\mathrm{m} \cdot \log \mathrm{v}+\log \mathrm{k}_{\mathrm{a}}
$$

con un buen grado de aproximación $\left(\mathrm{r}^{2} \sim 0.99\right)$ a los datos experimentales (v,F), Figura 1(b).

La misma metodología puede aplicarse al estudio de la influencia del avance sobre la fuerza de corte para las distintas velocidades de corte aplicadas. En la Figura 2(a) se muestra la evolución de la fuerza de corte con el avance, para diferentes velocidades de corte impuestas. La representación en el plano logarítmico, Figura 2(b), induce a proponer un modelo en la forma,

$$
\mathrm{F}=\mathrm{k}_{\mathrm{v}} \cdot \mathrm{a}^{\mathrm{n}}
$$

en el que los valores de $\mathrm{k}_{\mathrm{v}} \mathrm{y} \mathrm{n}$ dependen de la velocidad de corte $\mathrm{y}$ se obtienen del ajuste en escala logarítmica, Figura 2(b), de los datos experimentales a una recta del tipo

$$
\log \mathrm{F}=\mathrm{n} \cdot \log \mathrm{a}+\log \mathrm{k}_{\mathrm{v}}
$$

Como se aprecia en la Figura 2(b), los datos experimentales $(\mathrm{a}, \mathrm{F})$ presentan un buen ajuste $\left(\mathrm{r}^{2} \sim 0.99\right)$ a la recta de la ecuación (6).

De acuerdo con lo anteriormente expuesto, las relaciones marginales $\mathrm{F}(\mathrm{v})$ y $\mathrm{F}(\mathrm{a})$ dependen, respectivamente, de a y v. De esta forma, en las condiciones de trabajo empleadas, para el estudio de la fuerza de corte puede sugerirse el empleo de un modelo $\mathrm{F}(\mathrm{v}, \mathrm{a})$ que combine las dos relaciones potenciales, en la forma:

$$
\mathrm{F}=\mathrm{C} \cdot \mathrm{v}^{\mathrm{x}} \cdot \mathrm{a}^{\mathrm{y}}
$$

Como se deduce de las ecuaciones (3) y (5), en esta expresión C, $\mathrm{x}$ e y dependen de las condiciones de corte y son función de las variables impuestas. Gráficamente, la ecuación (7) es una superficie que puede generarse por el algoritmo de Coons [6] a partir de la interpolación de dos familias de curvas de Bézier dadas por las relaciones $\mathrm{F}(\mathrm{v})$ y F(a), Figura 3(a). La superficie así construida permite efectuar predicciones sobre el comportamiento de la fuerza de corte para distintas combi- naciones de v y a. Así, un plano paralelo al plano a-F define en la superficie una curva $\mathrm{F}(\mathrm{a})$ para un valor de la velocidad de corte, (ABCD en la Figura 3(a)) que predice la evolución de F como una función del avance para diferentes valores de la velocidad de corte aplicados. De forma similar, cuando se traza un plano paralelo al plano v-F, (EFGH en la Figura 3(a)) se obtiene la curva F(v) que determina el comportamiento de $\mathrm{F}$ en función de la velocidad de corte para los distintos avances aplicados.

Para determinar los valores de C, x e y, se efectúa una traslación a escala logarítmica de la ecuación (7)

$$
\log \mathrm{F}=\mathrm{x} \cdot \log \mathrm{v}+\mathrm{y} \cdot \log \mathrm{a}+\log \mathrm{C}
$$

Esta ecuación corresponde a un plano en el espacio (log a-log v-log F), Figura 3(b). En este espacio, los valores de C, x e y se pueden determinar por regresión multilineal de los datos experimentales:

$$
\mathrm{x}=-0.14
$$$$
\mathrm{y}=0.78
$$

Como puede observarse en la Figura 3(b), existe una buena aproximación entre los valores experimentalmente y los simulados, reflejado en los valores del error estándar y la dependencia marginal, Tabla I. En consecuencia, el modelo propuesto adopta la forma:

$$
\mathrm{F}=2.96 \cdot \mathrm{v}^{-0.14} \cdot \mathrm{a}^{0.78}
$$

Tabla I. Parámetros de ajuste multilineal de la ecuación (8)

\begin{tabular}{|c|c|c|}
\hline Parámetro & $\varepsilon_{0}$ & $\mathbf{r}^{2}$ \\
\hline $\mathrm{C}$ & 0.25 & 0.99 \\
\hline $\mathrm{x}$ & 0.02 & 0.89 \\
\hline $\mathrm{y}$ & 0.09 & 0.98 \\
\hline
\end{tabular}

\section{CONCLUSIONES}

La Fuerza de Corte, F, es uno de los parámetros que puede proporcionar una mayor información sobre un determinado proceso de mecanizado. Sin embargo, para su control se requiere el estudio de la dependencia con un elevado número de variables. Con objeto de 

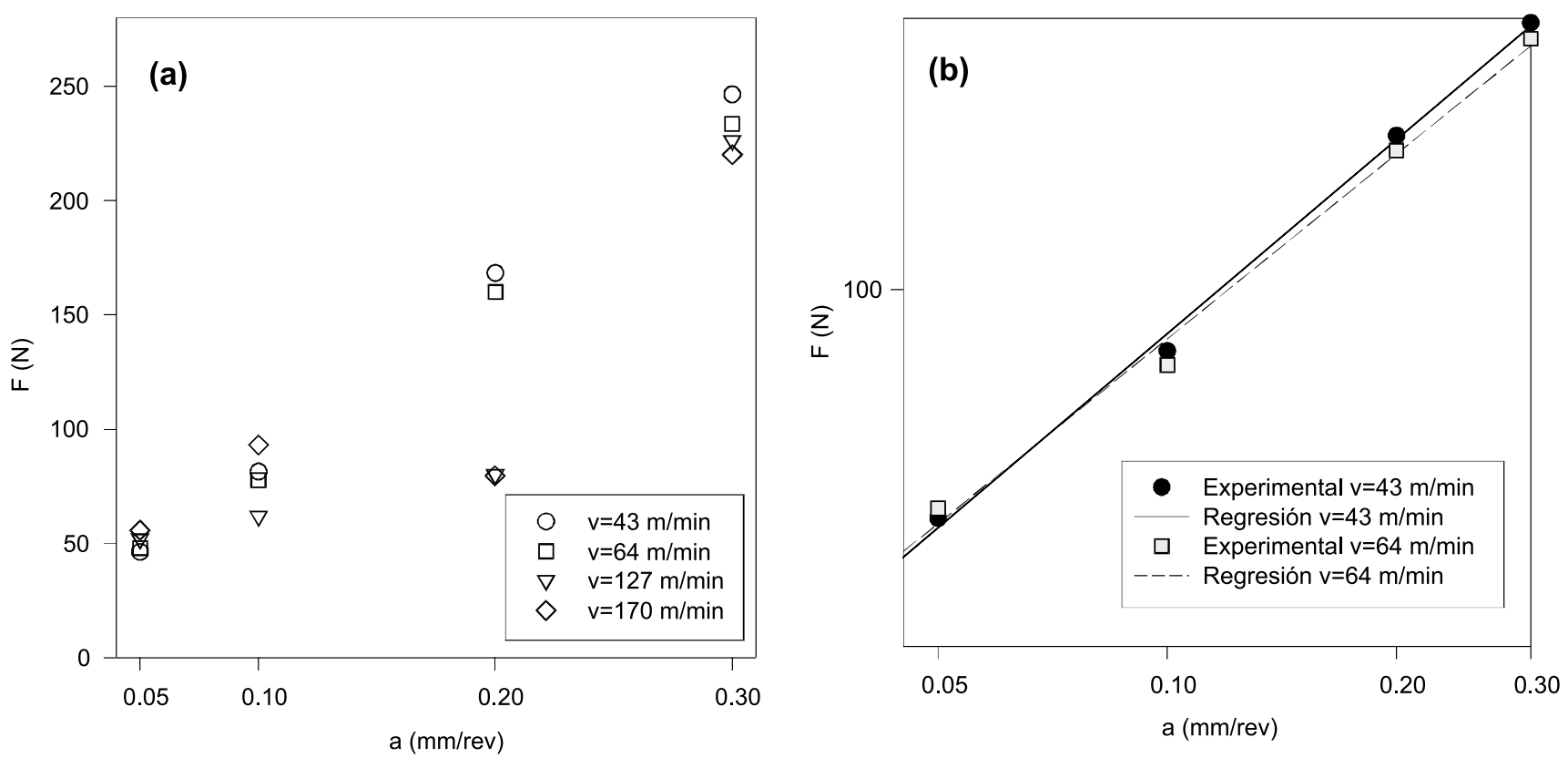

Figure 2. Evolución de la Fuerza de Corte con el avance para las distintas velocidades de corte aplicadas. (a) Escala Lineal. (b) Escala Logarítmica.

reducirlo, en este trabajo se ha efectuado un estudio de la fuerza de corte para un proceso concreto en el que sólo se han empleado como variables la velocidad de corte, $\mathrm{v}, \mathrm{y}$ el avance, $\mathrm{a}$.

En concreto se ha llevado a cabo un estudio experimental de la fuerza de corte en función de v y a, para el torneado en seco de la aleación AA2024. Inicialmente, se han establecido relaciones potenciales marginales $\mathrm{F}(\mathrm{a})$ y $\mathrm{F}(\mathrm{v})$. Dichas relaciones presentan respectivamente, una dependencia con la variable fijada, $\mathrm{v}$ y a. Como consecuencia de esto, se ha diseñado un modelo $\mathrm{F}(\mathrm{v}, \mathrm{a})$ que tiene un buen ajuste a los datos experimentales. Finalmente, el modelo propuesto puede predecir el comportamiento del sistema cuando se aplican diferentes valores devya.

\section{AGRADECIMIENTOS}

Este trabajo ha sido financiado por la Dirección General de Ciencia y Tecnología, Proyecto DPI2001-3747, por AIRBUS España S.L. y por la Junta de Andalucía.

\section{REFERENCIAS}

1. M.S. Carrilero, M. Marcos, "On the machinability of aluminium and aluminum alloys", J. Mech. Beh. Mater., 7[3] 179-193 (1996).

2. D. A. Axinte, W. Belluco, L. De Chiffre, "Evaluation of cutting force uncertainty components in turning" Int.J.Mach.Tool \& Manuf. 41 719-730 (2001).

3. M.S. Carrilero, J.M. Sánchez-Sola, J.M. González, J.P. Contreras, M. Marcos, "Cutting forces compatibility based on a plasticity model", Int. J. Mach. Tool \& Manuf. 42 559-565 (2002).

4. M.S. Carrilero, M. Marcos, V.M. Sánchez, “Feed, cutting speed and cutting force as machinability parameters of aluminium alloys", J. Mech. Beh. Mater. 7[3] 167-178 (1996).

5. M.S. Carrilero, M. Marcos, "Relaciones paramétricas en el mecanizado", Serv. Pub. UCA, (1994).

6. G. Farin, Curves and Surfaces for CAGD. Academic Press, (1993).

7. W.S. Lin,B.Y.Lee and C.L.Wu. "Modelling the surface roughness and cutting force for tunning", J. Mat. Proc. Tech. 108 [3] 286-293 (2001).
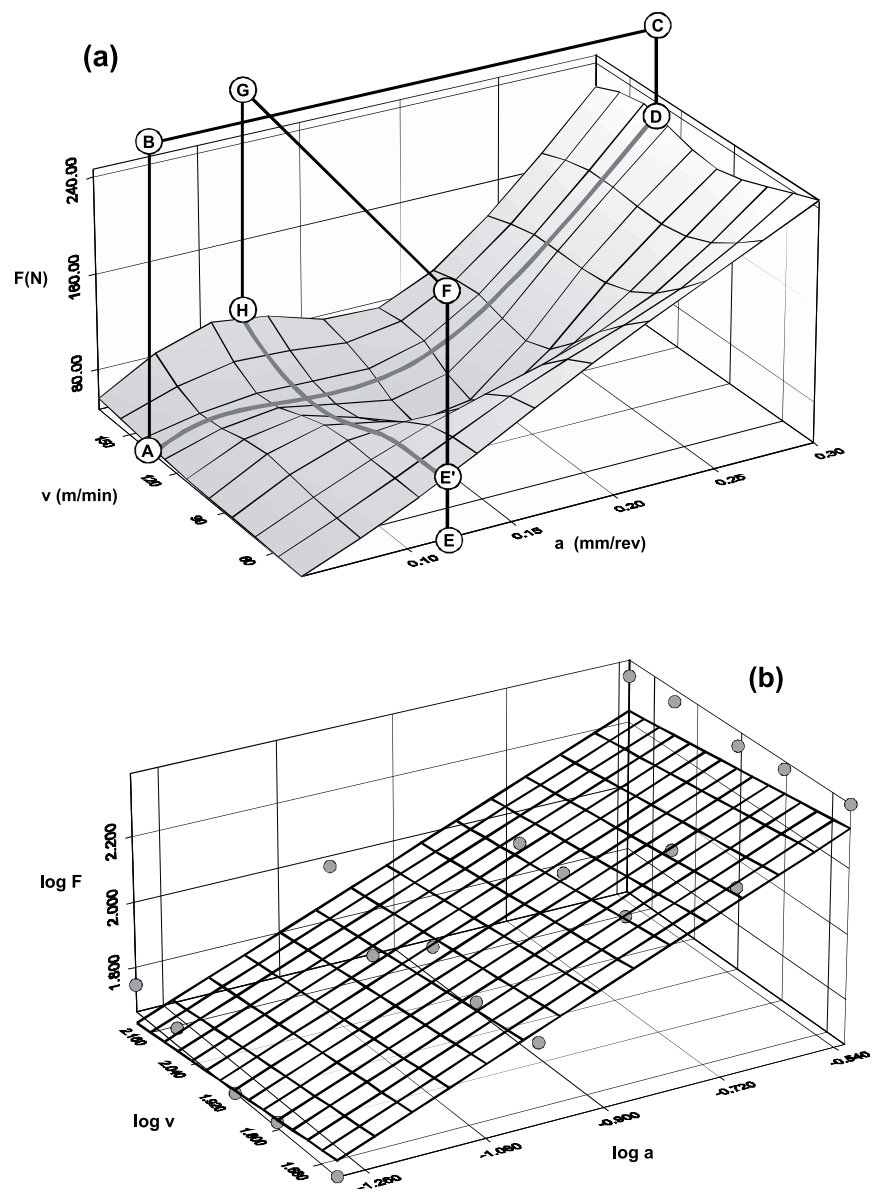

Figure 3. (a) Superficie Coons construida a partir de las familias de Curvas de Bézier F(v) y F(a). (b) Plano de regresión de F(v,a) en escala logarítmica. 